\title{
Editorial
}

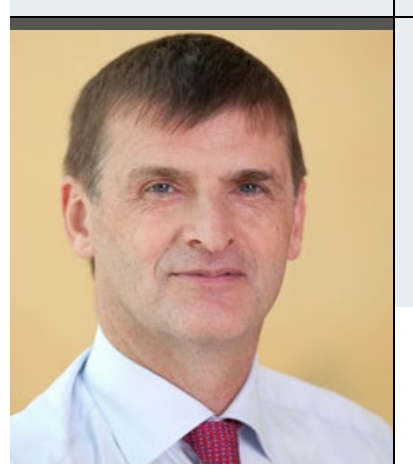

"Wir sind sehr gut ausgebildete Akademiker, die auf hohem

Niveau arbeiten, und sollten uns gegen die große Gleich-

macherei sowie Kommerzialisierung wehren."

\section{Parkinson-Syndrom: Wir wissen immer noch zu wenig!}

n den letzten Jahren hat die Wissenschaft sehr große Fortschritte erzielt. Wir haben das Gefühl, dass bereits viele Fragen beantwortet sind und wir beim Parkinson-Syndrom kurz vor einem Durchbruch stehen. Ich sehe dies jedoch sehr kritisch und postuliere, dass wir viel mehr Fragen als Antworten haben. Etliche unserer Studienergebnisse basieren auf Hypothesen und sind entsprechend kritisch zu werten. Die dramatische Zunahme der Studien und Publikationen korreliert nicht mit einem Erkenntniszuwachs. Stellenweise wäre es wünschenswert, man könnte Publikationen auch wieder löschen. Häufig ist die Fragestellung der Studie unsinnig und leider wird nur das Ergebnis betrachtet und auch zitiert. Seit der unüberschaubaren Zahl von frei zugänglichen Online-Journals greifen viele Kontrollmechanismen nicht mehr und fragwürdige Arbeiten tauchen sogar in PMC (PubMedCentral) auf.

\section{Wissenschaft und klinisches Wissen}

Wissenschaft und klinische Umsetzung stehen mittlerweile nur noch bedingt im Einklang und entwickeln sich eher auseinander. Wissenschaftler bemühen sich mit sehr großem Aufwand die Qualität auf hohem Niveau zu halten. Leitlinienkommissionen verwenden viel Zeit und Kraft, um das aktuelle Wissen zusammenzufassen. Vielerorts kümmern sich aber weder die Politik, noch die Kostenträger um die Ergebnisse oder die Meinungen. Selbst wenn man sich jahrelang mit einer Frage beschäftigt und die relevanten Daten kennt, hat dies gegenwärtig wenig Gewicht, da von offizieller Seite mit wenigen und auch unbegründeten Argumenten dagegen entschieden werden kann. Selbst erfahrene Kollegen aus unserem Fachgebiet fordern, dass in den Leitlinien ausgewiesene Wissenschaftler durch "neutrale" Personen ersetzt werden; dies mag politisch korrekt sein, führt jedoch zu einer unerträglichen Trivialisierung. Selbst wenn man sich sein ganzes be- rufliches Leben klinisch und wissenschaftlich mit dem Parkinson-Syndrom beschäftigt hat, wird man das Krankheitsbild und das vorhandene Wissen nicht überschauen können. Die meisten Fragen sind unbeantwortet und können aktuell nur mit dem besten verfügbaren klinischen Wissen beantwortet werden. Die Leitlinien versuchen das aktuelle Wissen zusammenzutragen, müssen aber viele Fragen unbeantwortet lassen.

\section{Wozu führt das und wie können wir dies positiv ändern?}

Viele Fragen in der Medizin sind nicht beantwortet und werden auch kurzfristig nicht gelöst. Wir müssen ständig weiter daran arbeiten und sollten unsere Grenzen auch erkennen und zugeben. Wir sollten uns aber auch gegen Scharlatane und Esoterik abgrenzen. Die Tatsache, dass wir eine Frage nicht beantworten oder ein Symptom nicht behandeln können, beweist nicht, dass andere unbewiesene Maßnahmen gleichwertig sind. Es ist auch nicht nachvollziehbar, dass wir unsere Therapie mit aufwändigen Studien beweisen müssen und die Kostenträger großzügig unbewiesene Alternativmedizin finanzieren. Es gibt nur eine Medizin, so wie es nur eine Mathematik, Physik oder Chemie gibt. Es ist auch ein riesiger Unterschied, ob ein erfahrener Parkinson-Spezialist oder eine auf diesem Gebiet unerfahrene Person eine Option diskutiert. Wenn beispielsweise ein Parkinson-Spezialist den Einsatz von Quetiapin bei der Parkinson-Psychose empfiehlt, da er jahrelange Erfahrung bei Hunderten von Patienten hat, ist dies die höchste verfügbare Evidenz und sollte akzeptiert werden, da es keine Studien gibt und auch absehbar nicht geben wird.

Wir brauchen mehr qualifizierte Studien und wir brauchen auch wieder mehr Respekt vor Wissen und Erfahrung. Wenn wir mit der Industrie Studien durchführen, werden wir schnell der Beeinflus- 


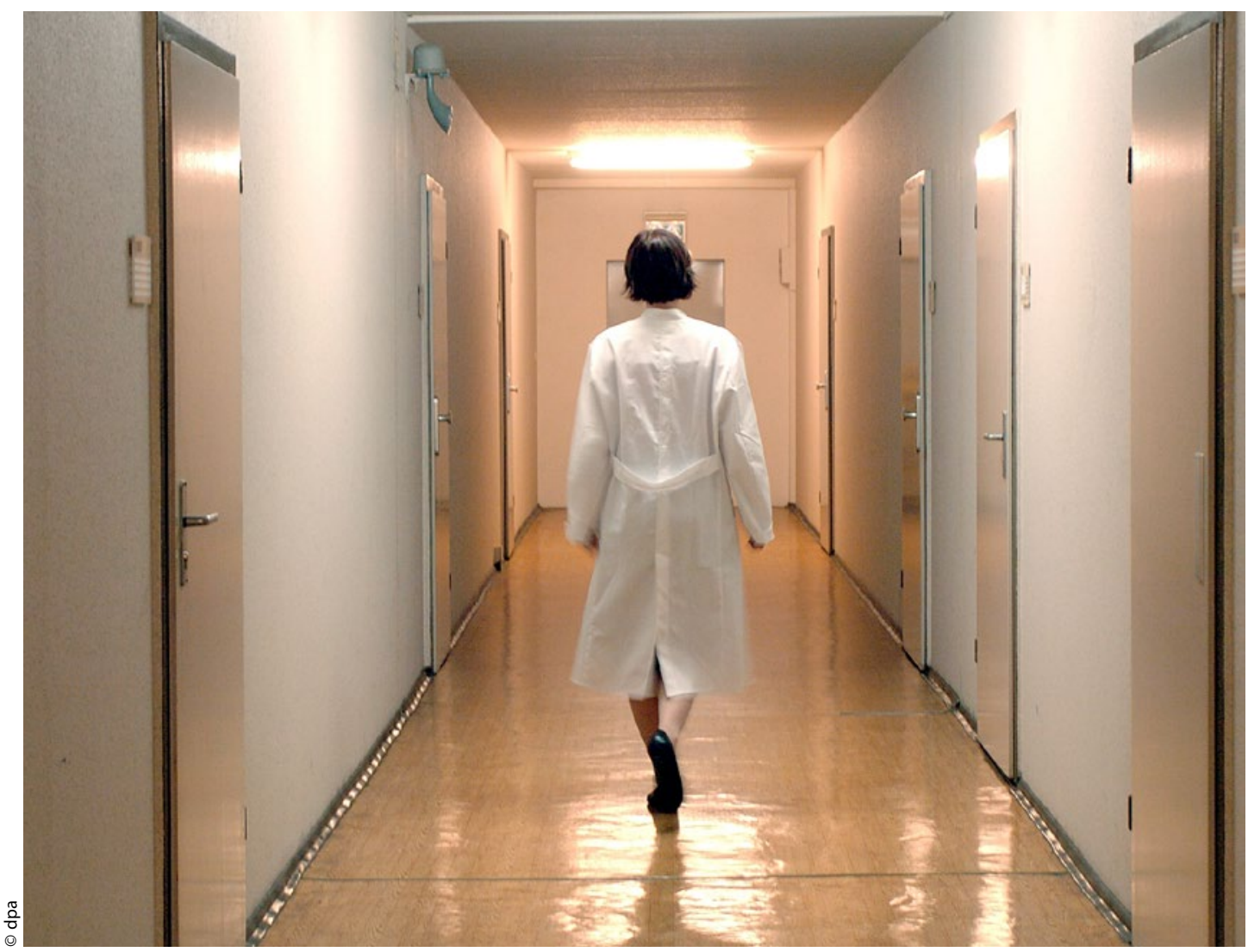

Wer wissenschaftlich interessiert ist, wird sich von der Klinik abwenden und andere Betätigungsfelder suchen, um Anerkennung zu finden.

sung und Bestechlichkeit bezichtigt. Sonstige Gelder für Studien gibt es aber zu wenig und Kostenträger unterstützen wissenschaftlichen Studien kaum. Früher wurden viele Studien von engagierten Kolleginnen und Kollegen in der Freizeit durchgeführt. Womit wir beim nächsten großen Problem wären: Uns fehlt der Nachwuchs sowie die Bereitschaft des Nachwuchses selbstlos zu arbeiten. Unser Nachwuchs ist sicherlich motiviert und engagiert, aber nicht masochistisch. Warum sollte ein junger Mensch jahrelang seine Freizeit im Labor oder mit Statistiken verbringen, damit er hinterher weder berufliche, noch gesellschaftliche oder finanzielle Anerkennung findet? In den Kliniken entscheiden nicht mehr die Chefärzte, sondern die Verwaltungen. Neue Medikamente werden nicht mehr unter klinischen und wissenschaftlichen Gesichtspunkten, sondern wirtschaftlichen Aspekten betrachtet. Dem Internet wird mehr geglaubt als dem erfahrenen Facharzt. Wer wissenschaftlich interessiert ist, wird sich von der Klinik fernhalten. Wer Karriere machen will, sucht sich andere Betätigungsfelder.

Wir sollten aber zumindest das letzte verbliebene Eiland verteidigen: die klinische Arbeit. Wenn ein sehr erfahrener, anerkannter Parkinson-Spezialist eine Therapie einsetzt, weil sie im Einzelfall indiziert ist und nichts dagegen spricht, sollte dies auch akzeptiert werden. Dies ist auf keinen Fall die Berechtigung dafür, dass wenig Erfahrene auch jeglichen Therapieversuch unternehmen dürfen. Wir sind sehr gut ausgebildete Akademiker, die auf hohem Niveau arbeiten - wie man auch am Beitrag von Professor Ebersbach in dieser Ausgabe sieht und sollten uns gegen die große Gleichmacherei sowie Kommerzialisierung wehren. In der klinischen Arbeit sind wir Ärzte anderen überlegen und dies wird auch so bleiben. Hier zählen Wissen und Erfahrung. Wir brauchen unsere Therapiefreiheit und viele klinische Studien, aber wir brauchen auch wieder mehr Respekt, von außen wie von innen.

Ihr

\section{Wolfgang Jost}

Prof. Dr. med. Wolfgang Jost

Parkinson-Klinik Wolfach, Kreuzbergstr. 12-24, 77709 Wolfach

E-Mail:w.jost@parkinson-klinik.de 